\title{
AVALIAÇÃO DOS EFEITOS DO PROCESSAMENTO SOBRE O POTENCIAL ANTIOXIDANTE DE NOVOS PRODUTOS
}

\author{
Gabriela Guedes Leite* \\ Brenda Nicolly Ribeiro Silva* \\ Elândia Aparecida dos Santos ** \\ Michelle Rosa Andrade Alves ** \\ Wiliam César Bento Régis ***
}

\begin{abstract}
RESUMO: Sabe-se da importância do consumo de alimentos com propriedades antioxidantes, pela constante exposição da população aos fatores que contribuem para o desenvolvimento de radicais livres e que consequentemente levam ao aumento no desenvolvimento de doenças crônicas no mundo moderno. Sendo assim, os produtos aqui descritos foram criados com a finalidade de terem alto teor antioxidante, mantendo suas características sensoriais. Este estudo objetivou analisar quais os tipos de processamento em que um alimento é submetido que preservarão 0 teor antioxidante do alimento pronto para consumo, foram analisados produtos desenvolvidos à base de pequi, açaí e cogumelo. Foi realizada revisão bibliográfica sobre o efeito dos métodos de cocção nos produtos desenvolvidos no Laboratório de Técnica Dietética da PUC - Minas Gerais. Observou-se que o método de cocção (sendo o maior interferente, os que empregam calor úmido) e tempo em que os alimentos ficam expostos a elevadas temperaturas influenciam consideravelmente 0 teor de compostos antioxidantes nos mesmos. Sendo assim, métodos em que não há o emprego de calor úmido e/ou que são menos expostos ao binômio tempo/temperatura, são os mais viáveis e recomendados para preservar os compostos antioxidantes. Podendo ainda citar como o melhor método de conservação das propriedades nutricionais, a liofilização.
\end{abstract}

PALAVRAS-CHAVE: Antioxidantes; Cocção; Pequi; Açaí; Agaricus.

\section{PROCESSING EFFECTS ON THE ANTIOXIDANT CAPACITY OF NEW PRODUCTS}

ABSTRACT: Everyone is aware of the importance of food with antioxidant properties due to the constant exposure of the population to factors that contribute towards the development of free radicals and which increase the development of chronic disease worldwide. The products mentioned in current analysis, based on pequi, açai and mushrooms, aim at high antioxidant rates with original sensorial characteristics. Current analysis surveys the types of processes which a type of food undergoes for the preservation of its antioxidant rates for consumption. A bibliographical survey was undertaken on cooking methods for products developed by the Laboratory for Dietetic Technique of the PUC, Belo Horizonte MG Brazil. The cooking method (the greatest interfering factor due to humid heat) and time in which the foods are exposed at high temperatures greatly affect their antioxidant rates. Methods without humid heat and/or those least exposed to time/temperature are the most viable and thus recommended for the preservation of antioxidant compounds. Lyophilization is the best conservation method for nutrition factors.

KEYWORDS: Antioxidants; Cooking; Pequi; Açaí; Agaricus.

\footnotetext{
Acadêmica de Nutrição da Pontifícia Universidade Católica de Minas Gerais (PUC-Minas), Brasil.

* Nutricionista ; Pontifícia Universidade Católica de Minas Gerais (PUC-Minas), Brasil.

*** Departamento de Nutrição Pontifícia Universidade Católica de Minas Gerais (PUC-Minas), Brasil.

**** Docente no Programa de Pós-Graduação em Biologia de Vertebrados na Pontifícia Universidade Católica de Minas Gerais (PUC Minas), Programa de Pós-Graduação em Nutrição e Saúde da Universidade Federal de Minas Gerais (UFMG) e no Programa de Pós-Graduação em Medicina Tropical e Infectologia da Universidade Federal de Minas Gerais (UFMG), Brasil.
} 


\section{INTRODUÇÃO}

Diante de tantos fatores que contribuem para o desenvolvimento de doenças crônicas no mundo moderno, os antioxidantes são compostos que deveriam estar presentes na alimentação da população diariamente.

Sabe-se que existem vários tipos de antioxidantes, tais como vitaminas, minerais, compostos fenólicos, pigmentos naturais e sintéticos, e que cada um pode ser afetado por alguns fatores, como pH, luz, temperatura, tempo de cocção, meio lipídico, exposição ao ar livre, entre outros. Podendo ainda, dependendo da interação, ter efeito indesejado, transformando-se em pró-oxidantes, que são moléculas exógenas ou endógenas que induzem ao estresse oxidativo, ocasionando efeito contrário ao que se espera. A vitamina $C$, por exemplo, pode ter atividade tanto antioxidante, quando reduz peróxido de hidrogênio, quanto pró-oxidante em casos de altas doses, pois 0 ácido ascórbico é um facilitador da reação de Fenton (se expressa na redução de peróxido de hidrogênio $\left(\mathrm{H}_{2} \mathrm{O}_{2}\right)$ pelo $\mathrm{Cu}^{+}$e $\mathrm{Fe}^{2+}$ dando origem ao radical hidroxila), pois é capaz de reduzir os metais de transição essenciais à ocorrência desta reação. A vitamina $E$ pode ter efeito pró-oxidante caso não haja concentrações equivalentes de outros antioxidantes para regenerar o radical -tocoferila a -tocoferol (DINIZ, 2015).

0 uso de compostos antioxidantes naturais é um dos mecanismos de defesa mais importantes contra os radicais livres. Pequi (Caryocar brasiliense), açaí (Euterpe oleracea) e cogumelo (Agaricus BlazeI) são alimentos reconhecidos pelo seu alto potencial antioxidante.

0 pequi é um fruto com elevado teor antioxidante, em 100 gramas da polpa do fruto possui $209 \mathrm{mg}$ de fenólicos totais e 7,25 mg de carotenoides totais, sendo os principais carotenoides presentes a violanxantina, luteína e zeaxantina. Com resultados maiores de fenólicos totais do que a maioria das frutas consideradas com elevado teor antioxidante consumidas no Brasil, como o açaí (Euterpe oleracea); goiaba (Psidiumguayava); morango (Gingo biloba); abacaxi (Ananas sativa); graviola (Anona muricato) e maracujá (Passiflora edulis), e apresentando teor de fenólicos total menor apenas que a acerola (Malpighia glabra) e a manga (Mangifera indica) (LIMA et al., 2007). 0 estudo feito por Regis et al., (2015) apresentou resultados relevantes, mostrando que a casca do pequi, parte geralmente desprezada, é onde se encontra a maior quantidade de compostos fenólicos $(48,27 \mathrm{mg} / \mu \mathrm{g})$, quando comparada à polpa $(0,038 \mathrm{mg} / \mu \mathrm{g})$, ressaltando a importância de se utilizar 0 fruto integralmente, dando possibilidades a se criar novas receitas com alto valor nutricional.

0 açaí (Euterpe oleracea) é um fruto pequeno, arredondado e de cor roxa escura em função da presença de antocianinas, típico do norte do Brasil, é utilizado na forma de sorvetes, cremes, iogurte e licores (DINIZ, 2006). 0 fruto contém quantidades significativas de uma classe distinta de flavonoides, as antocianinas, 0 que 0 atribui propriedades antioxidantes, como lipídios, proteínas, fibras, minerais $(\mathrm{Mn}, \mathrm{Cu}, \mathrm{Cr}, \mathrm{B})$ e vitaminas (BERNAUD; FUNCHAL, 2011), além de compostos fenólicos, que também são compostos antioxidantes. (SANTOS et al., 2008).

Os cogumelos são considerados alimentos importantes para a saúde humana, apresentando em sua composição baixo teor energético e elevadas quantidades de minerais, aminoácidos essenciais, vitaminas e fibras. 0 basidiocarpo do Agaricus blazei contém $40-45 \%$ de proteínas, $38-45 \%$ de carboidratos, $6-8 \%$ de fibras, $5-7 \%$ de minerais e 3-5\% de gordura, valores baseados em relação à matéria seca, além de possuir ainda vitamina B1, B2 e niacina, e também grandes quantidades de minerais como potássio e cálcio. É fonte ainda de probióticos como quitina, hemicelulose, e -glucanas, mananas, galactanas e xilanas (SANTOS, 2013).

Sendo assim, o presente estudo avaliou como os métodos de cocção podem alterar a quantidade total de antioxidantes em produtos desenvolvidos à base de pequi (Caryocar brasiliense), açaí (Euterpe oleracea) e cogumelo (Agaricus Blazel) e, o quanto se consegue controlar esta perda, para resultados mais 
significativos na preservação destes compostos em produtos feitos a partir de alimentos com reconhecido potencial antioxidante.

\section{METODOLOGIA}

Foram avaliados produtos desenvolvidos no Laboratório de Técnica Dietética da Pontifícia Universidade Católicas de Minas Gerais - Unidade Barreiro, por alunos da universidade, que continham em sua composição ingredientes com reconhecido potencial antioxidante: açaí, cogumelo ou pequi. Todas as receitas foram enriquecidas com um dos ingredientes - açaí, cogumelo ou pequi - e foram comparadas à receita padrão. Os produtos criados e avaliados no laboratório em relação a $A A$ foram: cookie diet de chocolate enriquecido com cogumelo, desenvolvido por Maia et al., (2013); bolo de creme de milho enriquecido com cogumelo, desenvolvido por Figueiredo et al., (2014); pão de queijo enriquecido com cogumelo, desenvolvido por Pereira et al., (2015); iogurte enriquecido com cogumelo, desenvolvido por Oliveira et al., (2012); cookie com farinha de semente de açaí, desenvolvido por Batista et al., (2012); cookie com extrato liofilizado da semente do açaí, desenvolvido por Almeida et al., (2013) e, macarrão de pequi, desenvolvido por Claudino et al., (2013).

\subsection{METODOLOGIA DE AVALIAÇÃO DA ATIVIDADE ANTIOXIDANTE (AA)}

0 método de extração utilizado para a determinação da $A A$ de todos os produtos foi 0 2,2-Diphenyl-1-picryl-hidrazil (DPPH), em que um radical livre em solução sofre redução quando em contato com substâncias antioxidantes (ROESLER, 2007), exceto para 0 macarrão de pequi que foi feita determinação de proteínas, carboidratos, compostos fenólicos, flavonoides e atividade antioxidante. Para a determinação de proteína utilizou-se 0 método Lowry, que "tem como princípio uma mistura contendo molibdato, tungstato e ácido fosfórico (reagente Folin-Ciocalteau), que se reduz quando reage com a proteína, na presença do catalisador cobre (II), e produz um composto com absorção máxima em 750 nm" (SANTOS, 2012).

\subsection{METODOLOGIA DE ANÁLISE SENSORIAL}

Para análise sensorial dos produtos desenvolvidos a partir do cogumelo foi entregue aos participantes um Termo de Consentimento Livre e Esclarecido (CAAE/CEP 0224.0.213.000-11) e uma ficha para avaliação dos aspectos sensoriais e intenção de compra. Duas amostras iguais de cada receita foram apresentadas, sendo que uma das amostras apresentava 0 produto como "produto integral". 0 objetivo era fazer um teste cego para avaliar qual o impacto da aceitação do produto apresentado como "enriquecido" com os produtos com potencial antioxidante.

Os participantes foram orientados a avaliar as características sensoriais (aparência, odor, sabor e textura) com base estruturada de 7 pontos, composta pelos extremos $1=$ gostei muitíssimo e $7=$ desgostei muitíssimo, em que de 1 a 3 demonstrava uma aceitação positiva, 4 uma aceitação neutra e de 5 a 7 uma aceitação negativa. Realizou-se uma avaliação de aceitação de compra dos produtos por meio de escala não estruturada de 5 pontos, com extremos, $1=$ certamente não compraria e $5=$ certamente compraria, em que 5 e 4 demonstrava intenção de compra positiva, 3 intenção de compra neutra, 2 e 1 intenção de compra negativa. As médias das notas apresentadas foram obtidas por meio da Anova, que é um método estatístico padrão para a avaliação de variância amostral.

Dados de análise sensorial não foram mostrados para os produtos desenvolvidos a partir de pequi e açaí.

\section{RESULTADOS}

\subsection{COOKIE DIET DE CHOCOLATE ENRIQUECIDO COM COGUMELO (AGARICUS BLAZEI)}


Foram criados cookies diets com diferentes tipos de chocolate (chocolate branco, ao leite $\mathrm{e}$ meio amargo) enriquecidos com Agaricus blazei. Os resultados apresentados foram favoráveis, todos os cookies assados enriquecidos com Agaricus blazei apresentaram aumento da $\mathrm{AA}$ em relação ao cru, provavelmente pelo processo de desidratação causado pelo calor seco.

Tabela 1. Porcentual de atividade antioxidante do produto Cookie Diet de Chocolate com Cogumelo (Agaricus blazei)

\begin{tabular}{|c|c|c|}
\hline & PREPARAÇÃO & $\%$ AA \\
\hline & $\begin{array}{l}\text { Cookie diet com chocolate branco } \\
\text { cru }\end{array}$ & 39,6 \\
\hline & $\begin{array}{l}\text { Cookie diet com chocolate branco } \\
\text { assado }\end{array}$ & 37,65 \\
\hline & $\begin{array}{l}\text { Cookie diet com Agaricus blazei e } \\
\text { chocolate branco cru }\end{array}$ & 85,8 \\
\hline & $\begin{array}{l}\text { Cookie diet com Agaricus blazei e } \\
\text { chocolate branco assado }\end{array}$ & 105,75 \\
\hline & $\begin{array}{l}\text { Cookie diet com chocolate ao leite } \\
\text { cru }\end{array}$ & 33 \\
\hline $\begin{array}{l}\text { Chocolate } \\
\text { enriquecido }\end{array}$ & $\begin{array}{l}\text { Cookie diet com chocolate ao leite } \\
\text { assado }\end{array}$ & 107 \\
\hline $\begin{array}{l}\text { Cogumelo } \\
\text { (Agaricus }\end{array}$ & $\begin{array}{l}\text { Cookie diet com Agaricus blazei e } \\
\text { chocolate ao leite cru }\end{array}$ & 105 \\
\hline & $\begin{array}{l}\text { Cookie diet com Agaricus blazei e } \\
\text { chocolate ao leite assado }\end{array}$ & 114 \\
\hline & $\begin{array}{l}\text { Cookie diet com chocolate meio } \\
\text { amargo cru }\end{array}$ & 62 \\
\hline & $\begin{array}{l}\text { Cookie diet com chocolate meio } \\
\text { amargo assado }\end{array}$ & 21,6 \\
\hline & $\begin{array}{l}\text { Cookie diet com Agaricus blazei e } \\
\text { chocolate meio amargo cru }\end{array}$ & 111 \\
\hline & $\begin{array}{l}\text { Cookie diet com Agaricus blazei e } \\
\text { chocolate meio amargo assado }\end{array}$ & 112,89 \\
\hline
\end{tabular}

Fonte: Adaptado de Maia et al. (2013).

Percebe-se que o cookie feito com chocolate branco apresentou níveis menores de $\mathrm{AA}$, isso se deve ao fato de que 0 chocolate branco é feito com a manteiga de cacau e não a massa em si, 0 que 0 faz obter menor teor de vitaminas e minerais quando comparado ao chocolate ao leite ou meio amargo. Os cookies assados feitos com chocolate meio amargo e chocolate ao leite enriquecido com Agaricus blazei apresentaram percentuais consideráveis de AA (112, $89 \%$ - 114\%), o cookie de chocolate branco apresentou $(105,75 \%)$ de AA, sendo o menor valor encontrado, isso porque 0 chocolate meio amargo e ao leite possuem elevado teor de vitaminas, principalmente vitamina A e tocoferol, além de polifenóis e flavonoides. Foi observado que o chocolate ao leite obteve 0 valor mais relevante de AA dentre as preparações.

\subsection{BOLO DE CREME DE MILHO ENRIQUECIDO COM COGUMELO (AGARICUS BLAZEI)}

Tabela 2. Porcentual de atividade antioxidante do produto Bolo de Cogumelo (Agaricus blazei)

\begin{tabular}{|c|c|c|}
\hline \multicolumn{2}{|c|}{ PREPARAÇÃO } & $\begin{array}{r}\% \\
\text { AA }\end{array}$ \\
\hline \multirow{6}{*}{$\begin{array}{c}\text { Bolo de } \\
\text { Creme } \\
\text { de Milho } \\
\text { enriquecido } \\
\text { com } \\
\text { Cogumelo } \\
\text { (Agaricus } \\
\text { blazei) }\end{array}$} & Bolo padrão cru & 53,7 \\
\hline & Bolo padrão assado & 51,8 \\
\hline & $\begin{array}{l}\text { Bolo enriquecido com Agaricus blazei } \\
15 \mathrm{~g} \text { cru }\end{array}$ & 79,7 \\
\hline & $\begin{array}{l}\text { Bolo enriquecido com Agaricus blazei } 15 \\
\text { g assado }\end{array}$ & 74,7 \\
\hline & $\begin{array}{l}\text { Bolo enriquecido com Agaricus blazei } \\
20 \mathrm{~g} \text { cru }\end{array}$ & 93,4 \\
\hline & $\begin{array}{l}\text { Bolo enriquecido com Agaricus blazei } 20 \\
\text { g assado }\end{array}$ & 89,2 \\
\hline
\end{tabular}

Fonte: Adaptado de Figueiredo et al. (2014)

A partir das análises feitas, pode-se observar que todas as amostras enriquecidas com Agaricus Blazei (15 g e $20 \mathrm{~g}$ ) apresentaram valores significantes de atividade antioxidante em relação às amostras do bolo padrão.

Observa-se que 0 produto assado teve diminuição da $A A$, possivelmente devido a fatores interferentes como a temperatura e tempo de cocção, pois os compostos fenólicos podem ser inativados quando expostos a altas temperaturas. 0 bolo isento de farinha de Agaricus blazei teve AA de 51,8\% (assado), esse fato pode ser pela presença de ingredientes 
como milho e ovo, que são ricos em carotenoides, luteína e zeaxantina, percussores de vitamina $A$. Apesar da massa enriquecida com $20 \mathrm{~g}$ de Agaricus blazei (assado) ter apresentado o maior teor de AA $(89,2 \%)$, os autores escolheram a amostra enriquecida com $15 \mathrm{~g}$ de Agaricus blazei (assado), que apresentou $74,7 \%$ de AA para realizar a análise sensorial, pois esta quantidade não alterou aspectos sensoriais e 0 custo para confecção foi menor.

\subsection{PÃO DE QUEIJO ENRIQUECIDO COM FARINHA DE COGUMELO (AGARICSUS BLAZEI)}

Tabela 3. Porcentual de atividade antioxidante do Pão de Queijo com farinha de Cogumelo (Agaricus blazei)

\begin{tabular}{|c|c|c|}
\hline & PREPARAÇÃO & $\% A A$ \\
\hline \multirow{6}{*}{$\begin{array}{l}\text { Pão de Queijo } \\
\text { enriquecido } \\
\text { com farinha } \\
\text { de Cogumelo } \\
\text { (Agaricus } \\
\text { blazei) }\end{array}$} & Pão de queijo padrão cru & 39,1 \\
\hline & Pão de queijo padrão assado & 46,4 \\
\hline & $\begin{array}{l}\text { Pão de queijo - 10g Agaricus } \\
\text { blazei cru }\end{array}$ & 87,8 \\
\hline & $\begin{array}{l}\text { Pão de queijo - } 10 \mathrm{~g} \text { Agaricus } \\
\text { blazei assado }\end{array}$ & 58,1 \\
\hline & $\begin{array}{l}\text { Pão de queijo - } 15 \mathrm{~g} \text { Agaricus } \\
\text { blazei cru }\end{array}$ & 92,6 \\
\hline & $\begin{array}{l}\text { Pão de queijo - } 15 \mathrm{~g} \text { Agaricus } \\
\text { blazei assado }\end{array}$ & 85,8 \\
\hline
\end{tabular}

Fonte: Adaptado de Pereira et al. (2015).

A amostra isenta de Agaricus blazei (assado) teve valor considerável de $A A(46,4 \%)$, podendo atribuir este resultado à presença de ingredientes como ovo $\mathrm{e}$ óleo, pois ambos possuem potencial antioxidante, por serem alimentos fontes de vitamina E. Os produtos crus tiveram maior teor de AA em comparação ao produto assado, fato que se justifica pelo tempo de cocção e de temperatura em que o produto foi submetido, pois altas temperaturas podem inativar compostos fenólicos.

\subsection{IOGURTE ENRIQUECIDO COM COGUMELO (AGARICUS BLAZEI)}

Foram selecionadas seis amostras para análise físico-química do iogurte enriquecido com cogumelo Agaricus blazei (Tabela 4). Todas as receitas foram enriquecidas com 20,75 g de cogumelo, cada amostra continha $20 \mathrm{~mL}$ de iogurte: $1^{\mathrm{a}}$ amostra: iogurte caseiro com cogumelo hidratado, $2^{\mathrm{a}}$ amostra: iogurte caseiro com polpa de morango e cogumelo Agaricus blazei, $3^{\mathrm{a}}$ amostra: iogurte caseiro com polpa de morango, $4^{\mathrm{a}}$ amostra: iogurte caseiro diet com cogumelo Agaricus blazei, $5^{\mathrm{a}}$ amostra: iogurte caseiro diet com polpa de maracujá e cogumelo Agaricus blazei e, $6^{\mathrm{a}}$ amostra: iogurte caseiro diet com polpa de maracujá.

Os autores optaram por analisar o teor de compostos fenólicos e flavonoides das preparações. Pode-se observar que 0 iogurte com polpa de morango enriquecido com cogumelo apresentou maior teor de compostos fenólicos $(1,69 \mathrm{mg} / \mathrm{mL})$ em relação ao iogurte sem adição do cogumelo $(1,59 \mathrm{mg} /$ $\mathrm{mL}$ ). Percebe-se que o morango é uma fruta rica em compostos fenólicos, pois o iogurte caseiro sem sabor enriquecido com cogumelo apresentou apenas 0,54 $\mathrm{mg} / \mathrm{mL}$ desses compostos, em média $33,96 \%$ do teor antioxidante quando comparado ao iogurte com polpa de morango.

Tabela 4. Teor de compostos fenólicos e flavonóides no logurte com cogumelo (Agaricus blazei)

\begin{tabular}{|c|c|c|c|}
\hline & EPARAÇÃO & $\begin{array}{l}\text { Compostos } \\
\text { Fenólicos }\end{array}$ & $\begin{array}{c}\text { Flavonóides } \\
\mathrm{mg} / \mathrm{mL}\end{array}$ \\
\hline & $\begin{array}{l}\text { logurte padrão } \\
\text { morango }\end{array}$ & $X$ & $X$ \\
\hline & $\begin{array}{l}\text { logurte caseiro }+ \\
\text { cogumelo hidratado }\end{array}$ & 0,54 & 0,14 \\
\hline logurte & $\begin{array}{l}\text { logurte caseiro + } \\
\text { polpa de morango + } \\
\text { cogumelo }\end{array}$ & 1,59 & 0,13 \\
\hline $\begin{array}{c}\text { com } \\
\text { Cogumelo }\end{array}$ & $\begin{array}{l}\text { logurte caseiro }+ \\
\text { polpa de morango }\end{array}$ & 1,69 & 0,07 \\
\hline & $\begin{array}{l}\text { logurte caseiro diet + } \\
\text { cogumelo }\end{array}$ & 0,85 & 0,38 \\
\hline & $\begin{array}{l}\text { logurte caseiro diet + } \\
\text { polpa de maracujá + } \\
\text { cogumelo }\end{array}$ & 0,41 & 0,27 \\
\hline & $\begin{array}{l}\text { logurte caseiro diet + } \\
\text { polpa de maracujá }\end{array}$ & 0,9 & 0,09 \\
\hline
\end{tabular}

Fonte: Adaptado de Oliveira et al. (2012). 
As amostras de iogurte diet com cogumelo Agaricus blazei e iogurte diet com polpa de maracujá enriquecido com cogumelo obtiveram bons resultados em relação aos flavonoides, $0,38 \mathrm{mg} / \mathrm{mL}$ e $0,27 \mathrm{mg} / \mathrm{mL}$, respectivamente, pela adição do cogumelo Agaricus blazei. As amostras que apresentaram resultados insatisfatórios (iogurte de morango com cogumelo para flavonoides e iogurte diet de maracujá com cogumelo para compostos fenólicos), provavelmente tiveram interferentes nos resultados, segundo o laboratório de análise físico-químicas da Universidade Federal de Minas Gerais (UFMG).

\subsection{COOKIE COM FARINHA DE SEMENTE DE AÇAÍ (EUTERPE OLERACEA)}

Foi elaborada receita de cookie integral padrão (Tabela 5) e para a adição da farinha de semente de açaí foram utilizadas três medidas em receitas diferentes, $30 \mathrm{~g}, 40 \mathrm{~g}$ e $45 \mathrm{~g}$. Todas as receitas tiveram a mesma base, porém em três receitas foi adicionada a farinha de semente de açaí. Todas as amostras apresentadas tiveram resultados consideráveis em relação ao teor de atividade antioxidantes, inclusive o cookie que não teve adição da farinha de semente de açaí, que pode ser explicado pelo fato de ter uva passas em sua composição, pois é um alimento que possui compostos fenólicos e antocianinas (OLIVEIRA; SANTANA, 2012).

Tabela 5. Porcentual de atividade antioxidante do produto Cookie com farinha de

\begin{tabular}{|c|c|c|}
\hline & PREPARAÇÃO & $\% A A$ \\
\hline \multirow{8}{*}{$\begin{array}{l}\text { Cookies } \\
\text { com } \\
\text { farinha de } \\
\text { semente } \\
\text { de Açaí } \\
\text { (Euterpe } \\
\text { oleracea) }\end{array}$} & Cookie padrão cru & 91,68 \\
\hline & Cookie padrão assado & 98,26 \\
\hline & $\begin{array}{l}\text { Cookie com farinha de semente de } \\
\text { Açaí cru - } 30 \mathrm{~g}\end{array}$ & 86,5 \\
\hline & $\begin{array}{l}\text { Cookie com farinha de semente de } \\
\text { Açaí assado - } 30 \mathrm{~g}\end{array}$ & 109,26 \\
\hline & $\begin{array}{l}\text { Cookie com farinha de semente de } \\
\text { Açaí cru - } 35 \mathrm{~g}\end{array}$ & 78,26 \\
\hline & $\begin{array}{l}\text { Cookie com farinha de semente de } \\
\text { Açaí assado }-35 \mathrm{~g}\end{array}$ & 109,77 \\
\hline & $\begin{array}{l}\text { Cookie com farinha de semente de } \\
\text { Açaí cru }-40 \mathrm{~g}\end{array}$ & 104,22 \\
\hline & $\begin{array}{l}\text { Cookie com farinha de semente de } \\
\text { Açaí assado - } 40 \mathrm{~g}\end{array}$ & 106,55 \\
\hline
\end{tabular}

Fonte: Adaptado de Batista et al. (2012).
0 cookie feito com $40 \mathrm{~g}$ de farinha de açaí teve maior $A A$, sendo $104 \%$ para a amostra crua, e 0 enriquecido com $35 \mathrm{~g}$ maior resultado para a receita assada, tendo AA de 109,77\%. Todas as amostras tiveram AA maior nas receitas com a massa assada, essa diferença pode ser atribuída à melhor eficiência na extração dos fenólicos após a cocção, corroborando com os estudo de Sultana et al, 2008, que mediram a quantidade de antioxidantes em hortaliças, onde observaram aumento na quantidade de compostos fenólicos em cenoura, ervilha e espinafre, submetidos à cocção em forno micro-ondas por 05 minutos.

\subsection{COOKIE COM EXTRATO LIOFILIZADO DA SE- MENTE DO AÇAÍ (EUTERPE OLERACEA)}

Foram desenvolvidas três receitas, uma receita padrão sem adição de extrato de açaí, uma receita com semente de açaí triturada e a última com extrato liofilizado de açaí. As amostras analisadas mostram que a receita com extrato liofilizado cru teve AA mais significante que o cookie de farinha de semente de açaí triturada e 0 cookie padrão, pela purificação da extração e liofilização da farinha da semente de açaí, pois a liofilização preserva grande parte do teor nutricional e qualidade sensorial do produto (SANTOS et al.,2008).

Tabela 6. Porcentual de atividade antioxidante do produto Cookie com extrato liofilizado da semente do Açaí (Euterpe oleracea)

\begin{tabular}{|c|c|c|}
\hline & PREPARAÇÃO & $\%$ AA \\
\hline \multirow{6}{*}{$\begin{array}{c}\text { Cookies } \\
\text { com extrato } \\
\text { liofilizado } \\
\text { da semente } \\
\text { do Açaí } \\
\text { (Euterpe } \\
\text { Oleracea) }\end{array}$} & Cookie massa padrão cru & 72,04 \\
\hline & Cookie massa padrão assado & 92,24 \\
\hline & $\begin{array}{l}\text { Cookie com semente de Açaí } \\
\text { triturada cru }\end{array}$ & 63,26 \\
\hline & $\begin{array}{l}\text { Cookie com semente de Açaí } \\
\text { triturada assado }\end{array}$ & 87,92 \\
\hline & $\begin{array}{l}\text { Cookie com extrato de Açaí liofilizado } \\
\text { cru }\end{array}$ & 122,2 \\
\hline & $\begin{array}{l}\text { Cookie com extrato de Açaí liofilizado } \\
\text { assado }\end{array}$ & 122,47 \\
\hline
\end{tabular}

Fonte: Adaptado de Almeida et al. (2013). 
Mesmo 0 cookie com massa padrão apresentou AA considerável, isso pode ser relacionado à receita ter em sua composição uvas passas. As uvas possuem alto teor de antocianinas em sua composição, pigmentos naturais que conferem a coloração arroxeada da fruta. Esses pigmentos estão presentes, principalmente na casca da uva, com exceção de alguns tipos, que também possuem a polpa pigmentada. Mesmo com a secagem do fruto, as antocianinas ainda são preservadas (SANTOS et al., 2010). As amostras analisadas mostram que a receita com extrato liofilizado teve AA mais significante que 0 cookie de farinha de semente de açaí triturada e o cookie padrão, pela purificação da extração e liofilização da farinha da semente de açaí, alimentos liofilizados são produtos com alto valor agregado por reter grande parte de seus nutrientes originais, uma vez que emprega baixas temperaturas em seu processamento.

\subsection{MACARRÃO DE PEQUI (CARYOCAR BRASILIENSE)}

Analisando a quantidade de flavonoides do macarrão de pequi em relação à massa comum, percebe-se que o macarrão de pequi apresenta uma quantidade quase três vezes maior de flavonoides, mas após o cozimento a massa de pequi apresentou perda considerável do mesmo, igualando-se à massa sem pequi, isso provavelmente pelo binômio tempo $x$ temperatura, mesmoosflavonoidessendo relativamente estáveis a alguns fatores como temperatura, oxigênio e $\mathrm{pH}$, podem ocorrer perdas durante 0 cozimento. As perdas podem ser maiores ou menores, dependendo do tipo de cozimento e do alimento.
Tabela 7. Quantidade de compostos fenólicos e de flavonóides (mg/L) no Macarrão de Pequi (Caryocar brasiliense)

\begin{tabular}{|c|c|c|c|}
\hline PREP & ARAÇÃO & $\begin{array}{l}\text { Compostos } \\
\text { Fenólicos mg/ }\end{array}$ & $\begin{array}{c}\text { Flavonóides } \\
\mathrm{mg} / \mathrm{mL}\end{array}$ \\
\hline & $\begin{array}{l}\text { Macarrão sem } \\
\text { pequi cru }\end{array}$ & 0 & 0,51 \\
\hline Macarrão & $\begin{array}{l}\text { Macarrão com } \\
\text { pequi cru }\end{array}$ & 0,33 & 1,54 \\
\hline $\begin{array}{c}\text { (Caryocar } \\
\text { brasiliense) }\end{array}$ & $\begin{array}{l}\text { Macarrão sem } \\
\text { pequi cozido }\end{array}$ & 3 & 0,12 \\
\hline & $\begin{array}{l}\text { Macarrão com } \\
\text { pequi cozido }\end{array}$ & 0,27 & 0,11 \\
\hline
\end{tabular}

Fonte: Adaptado de Claudino et al. (2013).

\section{DISCUSSÃO}

0 estudo de Tomas et al., (2016) mostrou que 0 processamento de geleia de amora preta levou a uma redução considerável de fenólicos totais $(88 \%)$, flavonoides totais $(89 \%)$, antocianina $(97 \%)$ e capacidade antioxidante $(88-93 \%) \quad(P<0,05)$. Roncero-Ramos et al. (2017) estudaram a influência de diferentes métodos de cocção na preservação de compostos antioxidantes em cogumelos. As perdas mais significativas de atividade antioxidante foram detectadas nos cogumelos que passaram por ebulição e fritura. Aqueles que foram grelhados e cozidos em forno micro-ondas tiveram resultados positivos, sendo estes os melhores métodos para conservação do valor nutricional de cogumelos. Além dos métodos de cocção, os diferentes métodos de conservação dos alimentos também podem influenciar 0 valor nutricional dos mesmos, sendo assim, há uma demanda crescente por frutos frescos e com processamento mínimo, pois esta condição não interfere no frescor e valor nutricional destes alimentos mais sensíveis. Cunha (2001) estudou a influência dos processos de pasteurização rápida (HTST) e ultra-high-temperature (UHT) sobre 0 conteúdo e estabilidade das vitaminas $\mathrm{A}, \mathrm{E}$ e $\mathrm{C}$ no leite. Não houve perda significativa de vitamina $A$ e $E$ em 
ambos os processos. A vitamina $\mathrm{C}$ apresentou perda de $20 \%$ para pasteurização rápida e, $25 \%$ quando submetida ultra-high-temperature. 0 congelamento é um método de conservação bastante utilizado, um dos mais eficientes para preservar as características sensoriais dos alimentos, porém seus benefícios podem ser comprometidos pelos efeitos prejudiciais ao teor nutricional causado ao produto no descongelamento, sendo sua severidade maior quanto mais for demorada a remoção do calor. Tavares et al. (1998) avaliaram 0 efeito do congelamento em câmara e túnel para conservação de acerola, o congelamento em câmara fria $\left(-16 \mathrm{a}-18^{\circ} \mathrm{C} / 48 \mathrm{~h}\right)$ apresentou maiores perdas de vitamina $\mathrm{C}$ do que 0 congelamento em túnel $\left(-72^{\circ} \mathrm{C} / 5\right.$ $\min )$.

Apesar de alguns resultados controversos sobre a perda de compostos antioxidantes após a cocção, em geral, os alimentos quando expostos a temperaturas mais baixas e por menor tempo durante a cocção, apresentam resultados menos negativos. Para compostos fenólicos e carotenoides, há uma dificuldade na extração em alimentos crus (principalmente vegetais), sendo este um agravante para a avaliação da real influência da cocção sobre os mesmos, pois alguns trabalhos mostraram mais eficiência na extração após cocção (CAMPOS et al, 2008).

Souza et al., (2013) avaliaram o melhor método de se armazenar o pequi sob congelamento sem que haja alterações nas características físicoquímicas do fruto, e concluíram que ao picar o fruto em lâminas e as embalar a vácuo, terá grande perda considerável em sua composição de carotenoides, e que a melhor forma de se armazenar o fruto congelado é sem extrair seu caroço envolto de plástico filme PVC, desta maneira sua AA se perderá muito pouco, concluíram ainda que 0 estado de maturação do fruto influencia no teor de carotenoides do fruto. Ainda sobre o pequi, Rodrigues et al., (2013) avaliaram 0 azeite de pequi submetido a aquecimento, e concluíram também que após 60 minutos de aquecimento observou-se diminuição acentuada dos carotenoides.

\section{CONCLUSÃO}

A partir da análise da AA das preparações, percebeu-se que formas de preparo, tempo de cocção, temperatura e interação entre compostos de uma preparação podem interferir consideravelmente na atividade destes compostos antioxidantes, fazendose necessário avaliar a forma de preparo, tempo de cocção, humidade e ingredientes de uma receita, de modo a manter as propriedades nutricionais ao final da preparação, sem que haja alteração nas características sensoriais. No geral, todos os tipos de cocção empregados nos diferentes alimentos desenvolvidos, aqui descritos, mostraram que 0 processamento de alimentos causa perdas nutricionais.

$\mathrm{Na}$ análise dos novos produtos foi possível observar que os alimentos que foram submetidos à liofilização são mais indicados para receitas submetidas à cocção, pois manteve consideravelmente mais alta a AA ao ser exposto em calor seco, conservando assim as propriedades antioxidantes do alimento, mostrando-se 0 melhor método de conservação da propriedade nutricional do alimento. Percebe-se ainda a importância de haver um controle das variáveis de coleta dos frutos, que influenciam diretamente em sua composição química, e que pode também ser fator determinante para 0 conteúdo antioxidante dos alimentos.

\section{REFERÊNCIAS}

ALMEIDA, G.G.S. et al. Criação de um novo produto: cookies com extrato liofilizado da semente do açaí. 2013. Trabalho de Conclusão de Curso (Nutrição) - Pontifícia Universidade Católica PUCMinas, Belo Horizonte, 2013.

BAETA, D. S. Avaliação de caracteristicas físicoquímicas e bioquímicas do pequi (Caryocar brasiliense Camb.) em suas diversas formas de armazenamento. 2013. 123f. Dissertação (Mestrado) - Universidade Estadual Paulista Júlio de Mesquita 
Filho, Instituto de Química de Araraquara, 2013. Disponível em: <http://hdl.handle.net/11449/88020>. Acesso em: 10 janeiro 2017.

BATISTA, A. M. R. et al. Criação de um novo produto: cookies com farinha de semente de açaí. Trabalho de Conclusão de Curso (Nutrição) - Pontifícia Universidade Católica PUCMinas, Belo Horizonte, 2013.

BERNAUD, F. S. R.; FUNCHAL, C.D.S. Atividade antioxidante do açaí. Nutrição Brasil, Porto Alegre, set./out. 2011.

CAMPOS, F.M.; MARTINO, H.S.D.; SABARENSE, C.M.; SANT'ANA, H.M. Estabilidade de compostos antioxidantes em hortaliças processadas: uma revisão. Alim. Nutr., Araraquara, v. 19, n. 4, 2008.

CASTR0,R.W.;BORGES,G.S.C.;GONZAGA,L.V.; RIBEIR0, D.H.B. Qualidade do preparado para bebida obtido a partir de polpa de juçara submetida ao tratamento térmico. Brazilian Journal of Food Technology, Campinas, v. 19, 2016. Disponível em: <http://www. scielo.br/scielo.php?script=sci_arttext\&pid=S198167232016000100411\&lang=pt $>$. Acesso em: 16 março 2017.

CLAUDINO, G.A.; PINHEIRO, G.C.S.; ALVES, M.R.A. Criação de novo produto: macarrão de pequi. Trabalho de Conclusão de Curso (Nutrição) - Pontifícia Universidade Católica PUCMinas, Belo Horizonte, 2013.

CUNHA, M. F. Revisão: leite UHT e o fenômeno de gelatinização. Boletim CEPPA, Paraná, v.19, n.2, p.341-352, 2001.

DINIZ, S. N. C. Vitaminas antioxidantes, carotenóides, polifenóis e envelhecimento. 2015. Disponível em: <https://estudogeral.sib.uc.pt/ bitstream/10316/31968/1/tese.pdf>. Acesso em: 10 janeiro 2017.

FIGUEIREDO, Á. M. et al. Criação de novos produtos: bolo de creme de milho enriquecido com cogumelo Agaricusblazei. Trabalho de Conclusão de Curso
(Nutrição) - Pontifícia Universidade Católica PUCMinas, Belo Horizonte, 2014.

LIMA, A.; SILVA, A. M. 0.; TRINDADE, R. A.; TORRES, R. P.; MANCINI-FILHO, J. Composição química e compostos bioativos presentes na polpa e na amêndoa do pequi (Caryocar brasiliense, Camb.). Rev. Bras. Frutic. Jaboticabal, v.29, n.3, 2007.

LOBO, A. C. M.; VELASQUE, L. F. L. Revisão de literatura sobre os efeitos terapêuticos do açaí e sua importância na alimentação. Biosaúde, Londrina, v. 18, n. 2, p.97-106, out. 2016. Disponível em: <http:// www.uel.br/revistas/uel/index.php/biosaude/article/ view/27624/20446>. Acesso em: 10 out. 2016

LOPES, T. J.; XAVIER, M. F.; QUADRI, M. G. N.; QUADRI, M. B. Antocianinas: uma breve revisão das características estruturais e da estabilidade. Rev. Bras. Agrociência, Pelotas, v.13, n.3, p. 291-297, jul./set, 2007.

MAIA, A. L. de A. et al. Criação de um novo produto: cookies diet enriquecido com cogumelo Agaricusblazei. Trabalho de Conclusão de Curso (Nutrição) - Pontifícia Universidade Católica PUCMinas, Belo Horizonte, 2013.

MORAES, K.; PINTO, L. A. A. Condições de secagem de uma pasta de anchoita modificada enzimaticamente na oxidação lipídica, lisina disponível e atividade antioxidante do produto. Ciência Rural, Santa Maria, v.43, n.3, p.530-536, mar, 2013. Disponível em: $\quad<$ http://www.scielo.br/scielo.php?script=sci_ arttext\&pid=S0103-84782013000300026\&lang=pt>. Acesso em: 16 março 2017.

MOREIRA, C. F. F.; LOPES, M. L. M.;VALENTE-MESQUITA, V.L. Impacto da estocagem sobre atividade antioxidante e teor de ácido ascórbico em sucos e refrescos de tangerina. Rev. Nutr., Campinas, v.25, n.6, p. 743752, nov./dez., 2012. Disponível em: <http://www. scielo.br/scielo.php?script=sci_arttext\&pid=S141552732012000600006\&lang=pt $>$. Acesso em: 16 março 2017.

OLIVEIRA, M. S. de; SANTANA, S. T. Criação de um novo produto: iogurte caseiro enriquecido com cogumelo 
Agaricus blazei. Trabalho de Conclusão de Curso (Nutrição- Pontifícia Universidade Católica PUCMinas, Belo Horizonte, 2012

PEREIRA, D. C. B. et al. Criação de novos produtos: pão de queijo enriquecido com cogumelo Agaricus blazei. Trabalho de Conclusão de Curso (Nutrição) - Pontifícia Universidade Católica PUCMinas, Belo Horizonte, 2015.

PEREIRA, E. A.; QUEIROZ, A. J. M.; FIGUEIRÊDO, R. M. F. de. Massa específica de polpa de açaí em função do teor de sólidos totais e da temperatura. Bras. eng. agríc. ambient. Campina Grande, v.6, n.3, set./dez. 2002.

REGIS, W. C. B.; SOUZA, M. R. R.; SILVEIRA, R. A. A. Comparação preliminar da composição química de diferentes partes do pequi (caryocar brasiliense) comercializado no vale do jequitinhonha e norte de minas gerais. Percurso acadêmico, v. 3, p. 269-279, 2013. Disponível em: <http://periodicos.pucminas. br/index.php/percursoacademico/article/view/5081>. Acesso em: 13 março 2017.

ROESLER, R. et al. Atividade antioxidante de frutas do cerrado. Ciênci. Tecnol. Aliment. Campinas, v.27, n.1, p. 53-60, jan./mar. 2007.

RONCERO-RAMOS, I. et al. Effect of different cooking methods on nutritional value and antioxidant activity of cultivated mushrooms. International Journal Of Food Sciences And Nutrition, v. 68, n. 3, p.287-297, 20 out. 2016. Disponível em: <http://dx.doi.org/10.1080/ 09637486.2016.1244662>. Acesso em: 16 nov. 2016

SANTOS, F. R.. Método de lowry: validação e estimativa do cálculo da incerteza. 2012. 63f. Dissertação (Mestrado) - Curso de Ciência dos Alimentos, Faculdade de Ciências Farmacêuticas da Universidade Estadual Paulista, Araraquara, 2012.

SANTOS, G. M.; MAIA, G. A.; SOUSA, P. H. M.; COSTA, J. M. C.; FIGUEIREDO, R. W.; PRAD0, G. M. Correlação entre atividade antioxidante e compostos bioativos de polpas comerciais de açaí (Euterpe oleracea ). ALAN, Caracas, v.58, n.2, jun. 2008.
SANTOS, P. R. Avaliação da capacidade antioxidante e composição fenólica de cogumelo Agaricus blazei Murrill. 2013. 31f. Trabalho de Conclusão de Curso (Graduação em farmácia) - Faculdade de Ceilândia da Universidade de Brasília, Brasília, 2013. Disponível em: <http://bdm.unb.br/bitstream/10483/7421/1/2013_ PolianaRodriguesDosSantos.pdf > . Acesso em: 20 jan. 2017.

SANTOS, P. et al. Avaliação físico-química e sensorial do pequi (Caryocar brasiliensis Camb.) submetido a desidratação. Revista Brasileira de Produtos Agroindustriais, Campina Grande, v.12, n.2, p.115123, 2010.

SOUZA, J. P.; ALVES, R. E.; BRITO, E. S.; NOGUEIRA, D. H.; LIMA, J. R. Estabilidade de produtos de pequi (Caryocar coriaceum Wittm) sob congelamento em diferentes tipos de embalagens. Rev. Bras. Frutic., Jaboticabal, São Paulo, v. 35, n. 4, p. 971-976, dez. 2013. Disponível em: <http://www.scielo. br/scielo.php?script=sci_arttext\&pid=S0100 29452013000400007\&lang=pt>. Acesso em: 19 março 2017.

SOUZA, M. A. C.; YUYAMA, L. K. 0.; AGUIAR, J. P. L.; PANTOJA, L. Suco de açaí (Euterpe oleracea Mart.): avaliação microbiológica, tratamento térmico e vida de prateleira. Acta Amaz, Manaus, v.36, n.4, out./dez. 2006.

SULTANA, B.; ANWAR, F.; IQBAL, S. Effect of different cooking methods on the antioxidant activity of some vegetables from Pakistan. Int. J. Food Sci. Technol., v.43, p.560-567, 2008.

TAVARES, J.C. et al. Métodos de congelamento na conservação da acerola (Malpighia emarginata DC.). Caatinga, Mossoró, v.11, p.71-74, 1998. Disponível em: <https://www.researchgate.net/profile/Francisco_ Bezerra_Neto/publication/277871896_METOD0S_ DE_CONGELAMENTO_NA_CONSERVACAO_ DA_ACEROLA_MALPIGHIA_EMARGINATA_DC/ links/5728f36b08ae2efbfdb7ec1f/METOD0SDE-CONGELAMENTO-NA-CONSERVACAO-DA- 
ACEROLA-MALPIGHIA-EMARGINATA-DC.pdf>. Acesso em: 15 março 2017.

TOMAS, M.; TOYDEMIR, G.; BOYACIOGLU, D.; HALL, R. D.; BEEKWILDER, J.; CAPANOGLU, E. Processing black mulberry into jam: effects on antioxidant potential and in vitro bioaccessibility. J. Sci. Food Agric., 2017. Doi:10.1002/jsfa.8152

VIEIRAL, A. P.; NICOLETIII, J. F.; TELISIII, V. R. N. Liofilização de fatias de abacaxi: avaliação da cinética de secagem e da qualidade do produto. Braz. J. Food Technol., Campinas, v.15, n.1, jan./mar. 2012.

Recebido em: 31 de março de 2017 Aceito em: 23 de maio de 2017 